\title{
Designing an e-democracy framework to enhance the Egyptian e- government
}

M. El-Sayed Wahed ${ }^{1}$, mahmoud El Arabawy ${ }^{2}$ and Esam M. El Gohary ${ }^{3}$

${ }^{1}$ Faculty of Computers and information, Suez Canal University, Egypt

email: mewahed@yahoo.com

${ }^{2}$ Faculty of Science, Suez Canal University, Egypt

email:marabawy@yahoo.com

${ }^{3}$ Faculty of Computer and Information Systems, Mansoura University ,Egypt

email: esam@minimax-soft.com

\section{Abstract}

E-democracy concept can enhance Egyptian participation in rule and it can move e-the Egyptian government to a new era. E-democracy concept can be applied in conjunction with database connection to transfer Egyptian e-government to transformation stage. This Paper concentrates mainly on designing an e-democracy framework using two case studies in e-government systems. As we will see system is web based application based .There are some figures that describe system architecture then each component well be explained. The framework technical aspects, analysis and objectives in establishing integrated e-government will be discussed.

\section{Council for Innovative Research}

Peer Review Research Publishing System

Journal: INTERNATIONAL JOURNAL OF COMPUTERS \& TECHNOLOGY

Vol 10, No 5

editor@cirworld.com

www.cirworld.com, member.cirworld.com 


\section{Introduction: What is E-democracy?}

"E-democracy is anything that governments do to facilitate greater participation in government using digital or electronic means. These initiatives can include e-forums, e-town hall meetings, e-consultations, e-referenda, e-voting, e-rule making, and other forms of e-participation. I believe we can also term it as any form of 'digital engagement'.[1]

E-democracy refers to the use of ICTs in democratic processes. It builds on opportunities provided by new ICTs, such as the internet, interactive digital television, and mobile communication systems, to increase publicparticipation in government decision-making.

E-Democracy will expand the range of opportunities for citizens to contribute to the debates and discussions on public policies, checks and balances on governments' activities and abuses of power and authority. In short, e-Democracy will facilitate the circulation of information, opinions, views and experiences, thus expanding the political arena and empowering the citizen to effectively participate at his/her own convenience and place in decisions which affect his/her life, livelihood and lifestyles. It will make politicians and bureaucrats more responsive to the needs and aspiration of the citizens, accountable and transparent, accessible and 'people-friendly'.

\subsection{The relation between E- government and E-democracy}

To adopt e-government means to transfer government activities into online forms. The goal of this transformation is the same as the goal of transferring a private company's activity to the Internet, i.e. to increase work effectiveness.

The concept of e-government is to facilitate citizens' access to a great amount of diverse information. For the public, egovernment means a simplification of their interaction with government thanks to Internet connections. The speed of the information exchange between bureaucrats and citizens is increasing dramatically. The main characteristic of advanced egovernment is interactivity.

Establishing e-government includes several stages.

- The first stage consists in the formation of different departmental and ministrial online sites, which carry only specific department information.

- At the second stage these federal and municipal sites become interactive. In some countries there exist special government pages where you can find answers to the most frequently asked questions. Some sites enable one to send email - which is the most accessible Internet service most often used by ordinary citizens.

- At the third stage, users are given the opportunity to take part in forums and opinion polls. The federal government and departments offer online services such as payment of fines, renewal of licences, registering one's car, moving house and so on.

- At the final stage (Transformation and Participation)all departments and government organizations in the country are brought together in a unified government portal, which offers a complex of different services for the population. The portal gives citizens an opportunity to take part in online discussions, comment on policy and legislation proposals and vote online. Ideally at this stage e-government may be used to further the means of democracy.(fig. 1)

fig.(1) The relation between e-government and e-democracy 


\section{2 paper importance}

the paper importance is increased as it introduce for e-participation models through e-democracy concept and egovernment applications, after the Egyptian two revolutions 25 january and 30 June the two revolutions has been raised because the absence of people participation in decision making.

The paper try to answer two questions first, "How can Egyptian e-government move from service based applications to eparticipation in order to solve the following problems" :

1-Lake of engagement between citizens and government.

2-Poor of traditional democracy to achieve (accountability-transparency-social participation- evaluation of service delivery)

3- Legacy systems and different technologies applied in many government agencies.

Second, how can Egyptian living abroad participate in their country decision making?

\section{Technical overview}

The idea of the proposed framework is mainly directly to solve the paper problems. This framework was applied by a designed system has a subsystems in the area of government.

There are some samples of the system screenshots for this system which importance only to try the two concepts of edemocracy and integration between legacy systems .First technical issues need to be addressed to help in understanding how the framework works.

\subsection{Service-Oriented Architecture (SOA)[2]}

Service-Oriented Architecture (SOA) is a new buzz word in the world of computing. SOA has evolved from age old computing concepts like modular/OOPS programming, Component based development(CBD) and distributed computing. It differentiates each module as a separate service. And a group of these services form parts of a Service-OrientedArchitecture of a system.

SOA is defined as "A set of components which can be invoked, and whose interface descriptions can be published and discovered"

\section{Service}

The corner stone of SOA is a Service. A Service runs as a separate entity and has specific functionality in the form of requests and responses. The underlying complexity of each service is not visible to the other services. They just need to know the request and response formats and query the service accordingly. The Service which responds to a request is called Service Provider and a Service which request for some data becomes a Service Consumer. Same Service can be a both Provider and Consumer of other services. Below picture shows one such relationship that could exist.

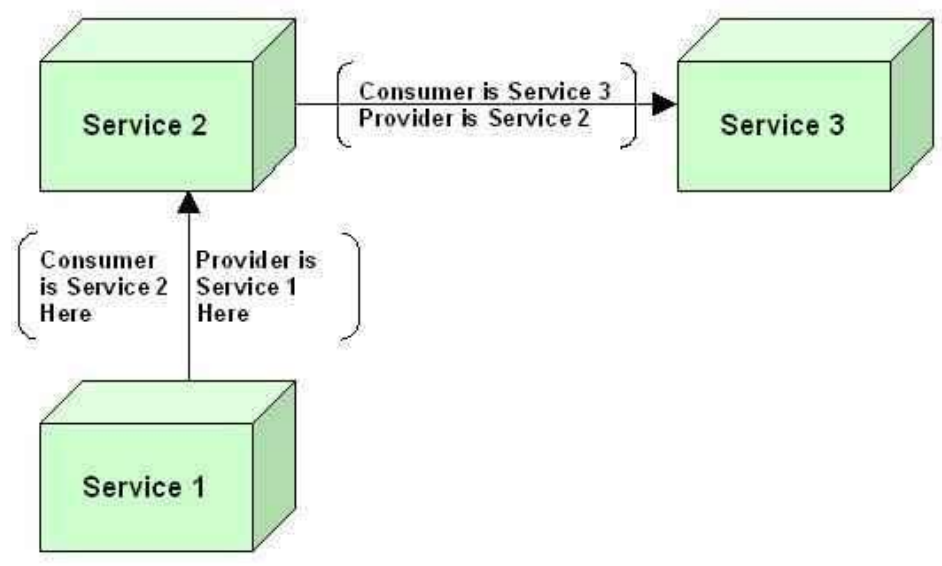

Fig(2) how services interact

Services have following essential features

\section{Visibility}

A Service Consumer should have prior knowledge of the access specifications to access a consumer. A Service provider has the right to reject the request from consumer if it feels that the consumer is not supposed to access it. These security settings are essential so that consumer access is preset for only valid users.

\section{Service Contracts}


A Service will be only useful if it comes with proper description(service contracts) of how a service can be accessed. The details of these descriptions will include

- $\quad$ Transport layer - Network protocol, Proxies, VPN's etc

- Services or tasks hosted by the service

- Security policies the consumer needs to follow to access provider.

- Data format of request and responses

\section{Major Advantages of SOA}

- Each service is loosely coupled or loosely independent of the other systems in the architecture.

- Services can sit different operating systems

- Each service can be written in different programming language.

- Complexity of each provider service is abstracted from the consumer service.

\subsection{SOAP (Simple Object Access Protocol)}

SOAP is a simple XML-based protocol to let applications exchange information between applications over HTTP.More simply: SOAP is a protocol for accessing a Web Service.

\section{What is SOAP?}

- $\quad$ SOAP stands for Simple Object Access Protocol

- SOAP is a communication protocol

- $\quad$ SOAP is for communication between applications

- $\quad$ SOAP is a format for sending messages

- SOAP communicates via Internet

- SOAP is platform independent

- $\quad$ SOAP is language independent

- SOAP is simple and extensible

- $\quad$ SOAP allows you to get around firewalls

- SOAP is a W3C recommendation

\section{Why SOAP?}

It is important for application development to allow Internet communication between programs.

Today's applications communicate using Remote Procedure Calls (RPC) between objects like DCOM and CORBA, but HTTP was not designed for this. RPC represents a compatibility and security problem; firewalls and proxy servers will normally block this kind of traffic.

A better way to communicate between applications is over HTTP, because HTTP is supported by all Internet browsers and servers. SOAP was created to accomplish this.

SOAP provides a way to communicate between applications running on different operating systems, with different technologies and programming languages.

\section{SOAP Building Blocks}

A SOAP message is an ordinary XML document containing the following elements:

- An Envelope element that identifies the XML document as a SOAP message

- A Header element that contains header information

- A Body element that contains call and response information

- A Fault element containing errors and status information

After this overview it is necessary to know that SOA was developed mainly for Business Process management and we will reuse it in G2G applications.

\section{The proposed e-democracy framework}

The system aims create and apply a general framework to enable e-Government services that is robust, flexible, scalable and integrated, and putting it into a practical case study. 
The following table shows how SOA can help to achieve the proposed features:

\subsection{Framework Features}

\begin{tabular}{ll}
\hline Robust & Anti Cheating and can work in abnormal conditions \\
Flexible & SOA enables flexibility by simply modifying web-services internal \\
logic without changing the public service interface. Web services \\
public interfaces apply loosely-coupled strategy between e- \\
Government applications. \\
SOA enables scalability by adding new web-services sand register \\
them into e-Government Service bus registry. New services may be \\
related to new e-Government applications or new feature of an \\
existing application. \\
Scalable \\
SOA enables decentralization of applications and services over \\
many several servers connected through the web which also \\
resolves the issue of scaling-up hardware severs. \\
e-Government service bus registry enables any e-Government \\
application to allocate and call any necessary methods offered by \\
another application's web-services. Using web services in SOA \\
keeps integration between e-Government easy and robust. \\
SOA uses SOAP protocol for communications between web- \\
Services based on XML. The protocol is simple text and supported \\
by all major development environments, platforms and operating \\
systems.
\end{tabular}

The above features of the framework can be achieved by applying the technology of Service Oriented Architecture (SOA) and employing XML and SOAP (Simple Object Application Protocol) as a main communication protocol. The following figure illustrate the e-democracy framework applied system .

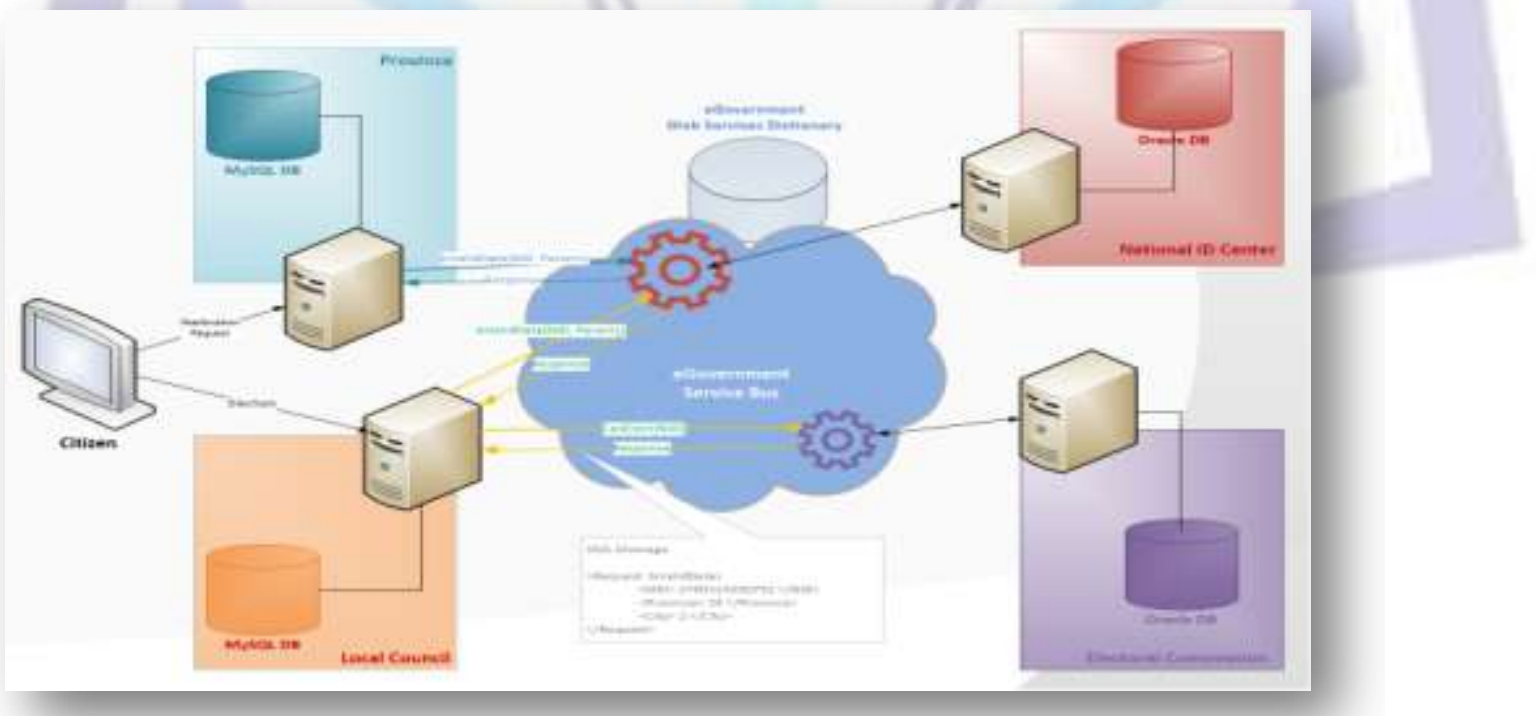

Fig (3) the integrated e-democracy framework

\section{Framework Components}

- Service bus registry

- Data repositories 
- Application web-service

- Authentication web service

- e-Government application

Framework components integrate together by SOAP calling messages between web services in order to process any given request. The following figure shows a high level activity diagram of the sequence of integration between the proposed framework components.

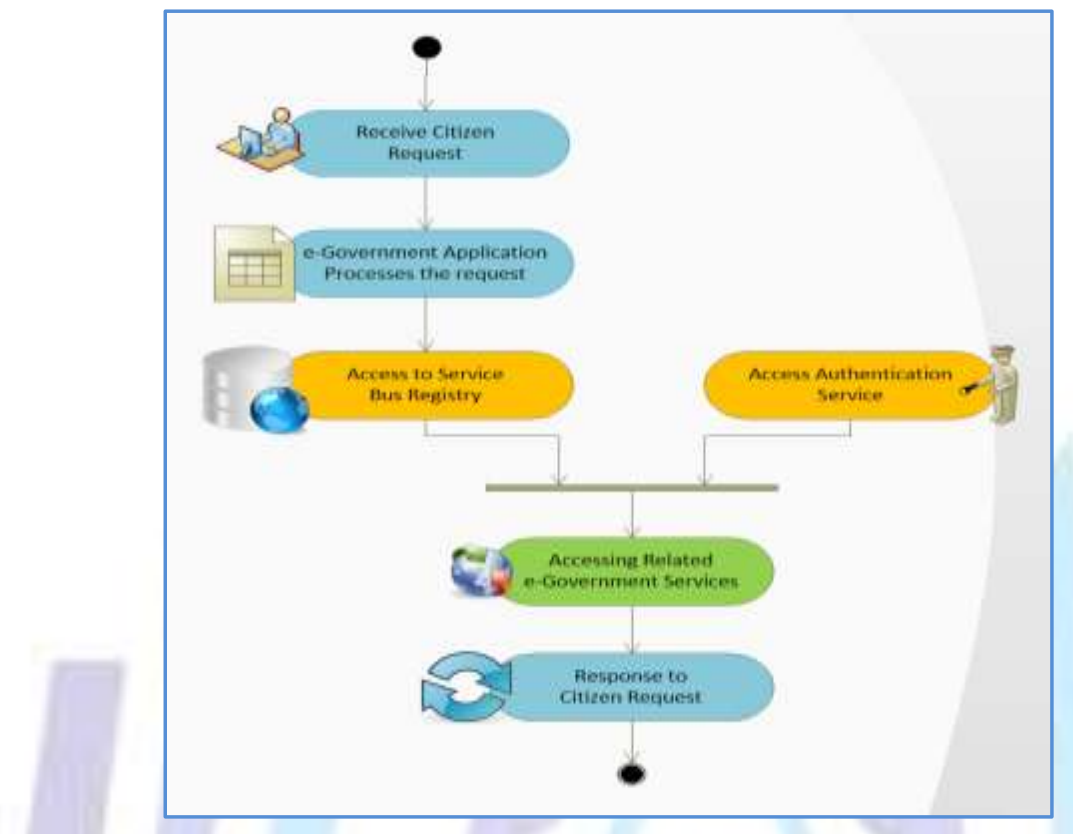

Fig (4) high level activity diagram

1. The process triggers by a citizen request sent to an e-Government application. The given request needs accessing data or services exposed by another e-Government application.

2. Service bus registry represents a central dictionary of all e-Government services available for access and integration. The registry provides all data needed to access the web service including
a. Service name
b. Publisher
c. Location
d. Service interface

3. The e-Government application must be authorized by the Authorization web-service in order to be able to communicate any e-Government web service.

4. The application accesses the appropriate e-Government web service using data given by the service bus registry and authentication token given by the authentication service.

5. The application receives response from the web-service, integrates it into process and sends the appropriate response to the user.

\section{Case Study 1: Local Multiplicity e-Voting}

In this case an e-Government application is created based on the proposed framework in order to offer a web portal of Ismaillia Local municipality. The web portal aims to enable and facilitate interaction between citizens and the local municipality of Ismaillia. Citizens are able to join some e-Voting services for the following use-cases.

1. UC1: Vote to select multiplicity members from a list of candidates.

2. UC2: Vote to approve/disapprove the yearly budget of the province.

3. UC3: Vote to approve/disapprove critical local decisions.

Use case 2 and 3 are two types of e-participation applications to improve the citizen's access to information and public service and maintain the citizen in decision making process.

The following figure shows the related activity diagram which represents the e-Voting process in the local multiplicity application. 


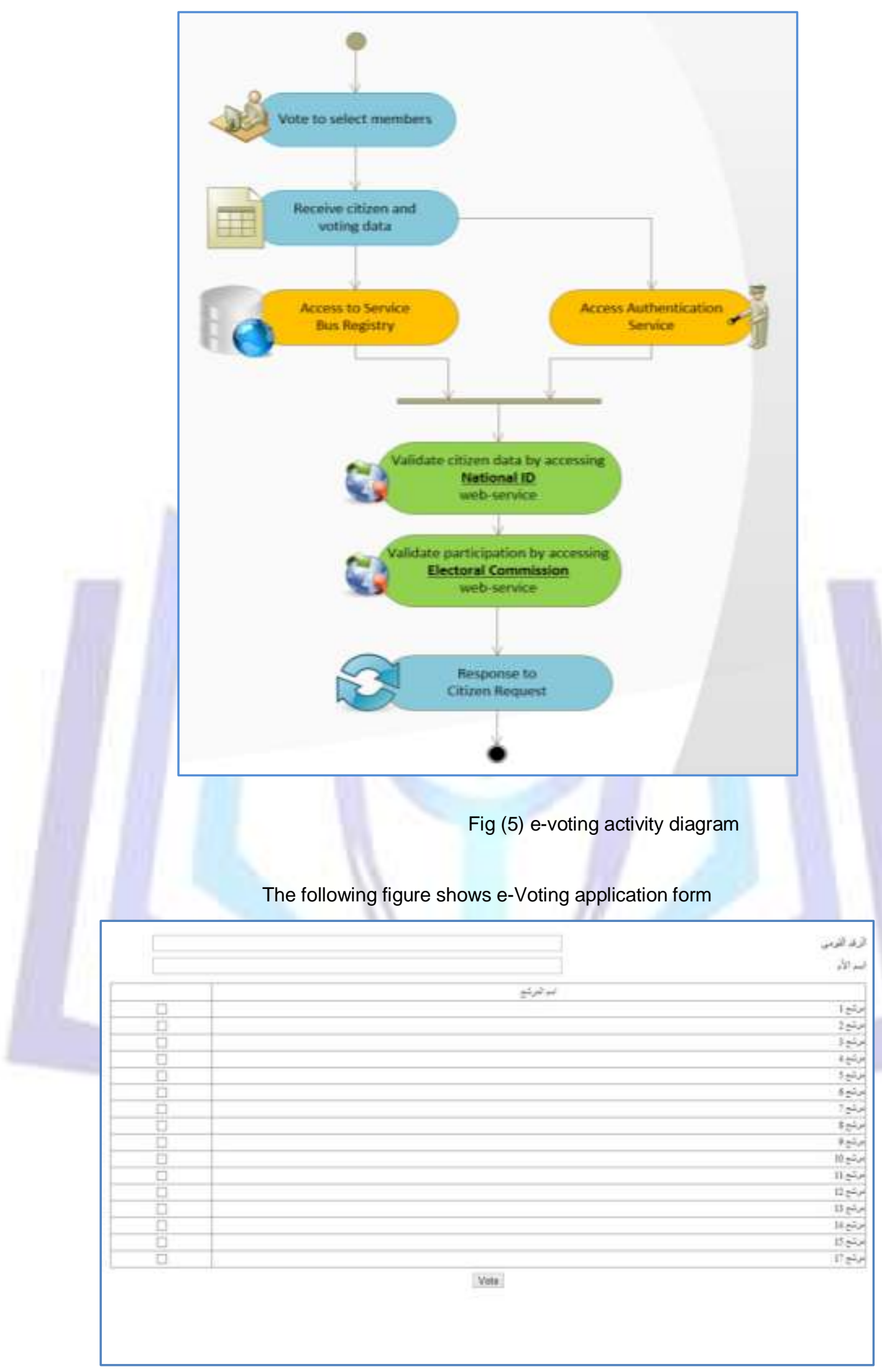

Fig (6) e-vot screen in the application

e-Voting application represents an interface of the e-Voting database and communicate to NID web service to validate voter data as shown in the following ERD which is independent to the different database types. 


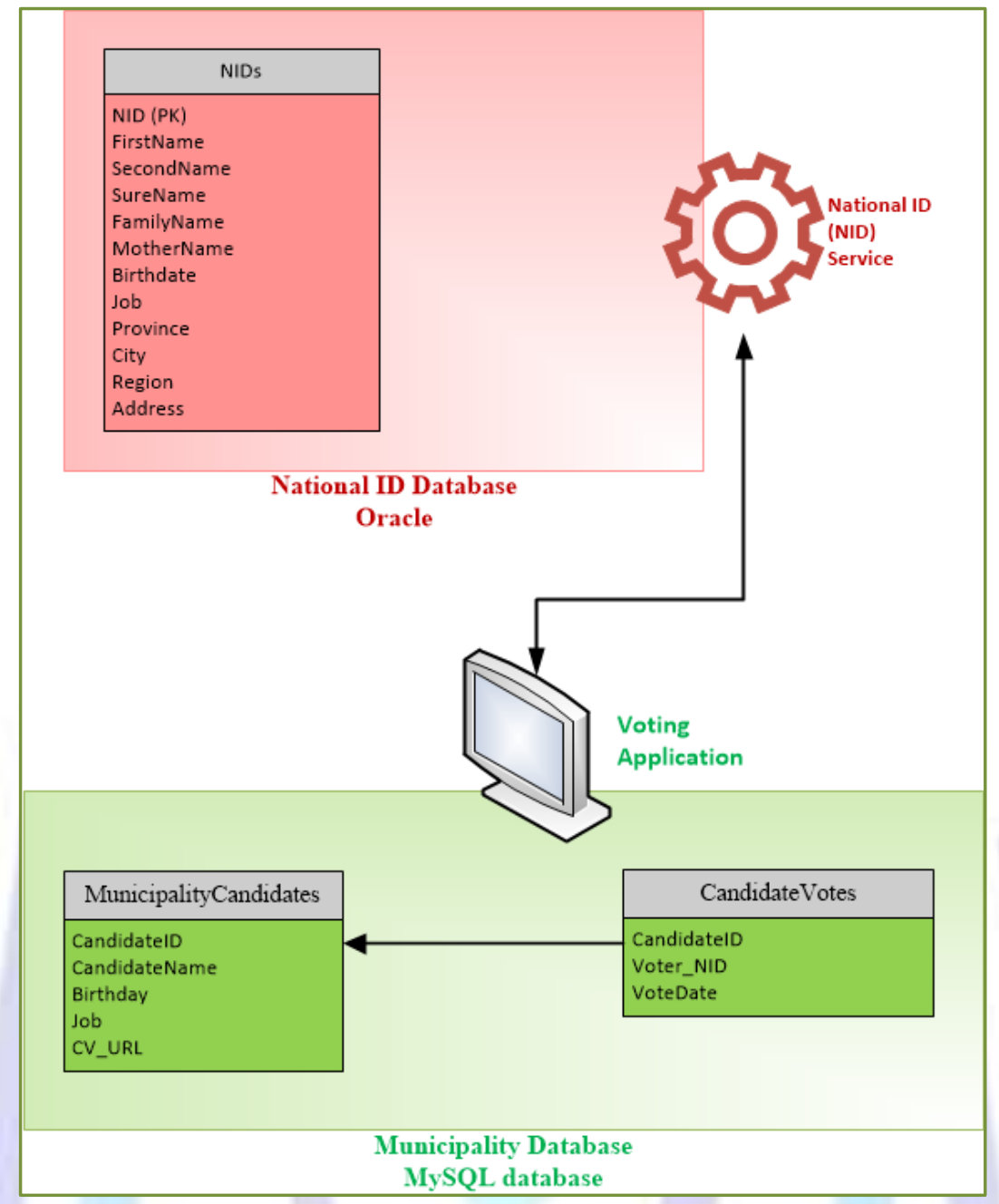

Fig. (7) applied system for the framework ERD

The system is able to integrate heterogeneous systems using SOA methodology and XML as communication protocol as in figure 8.

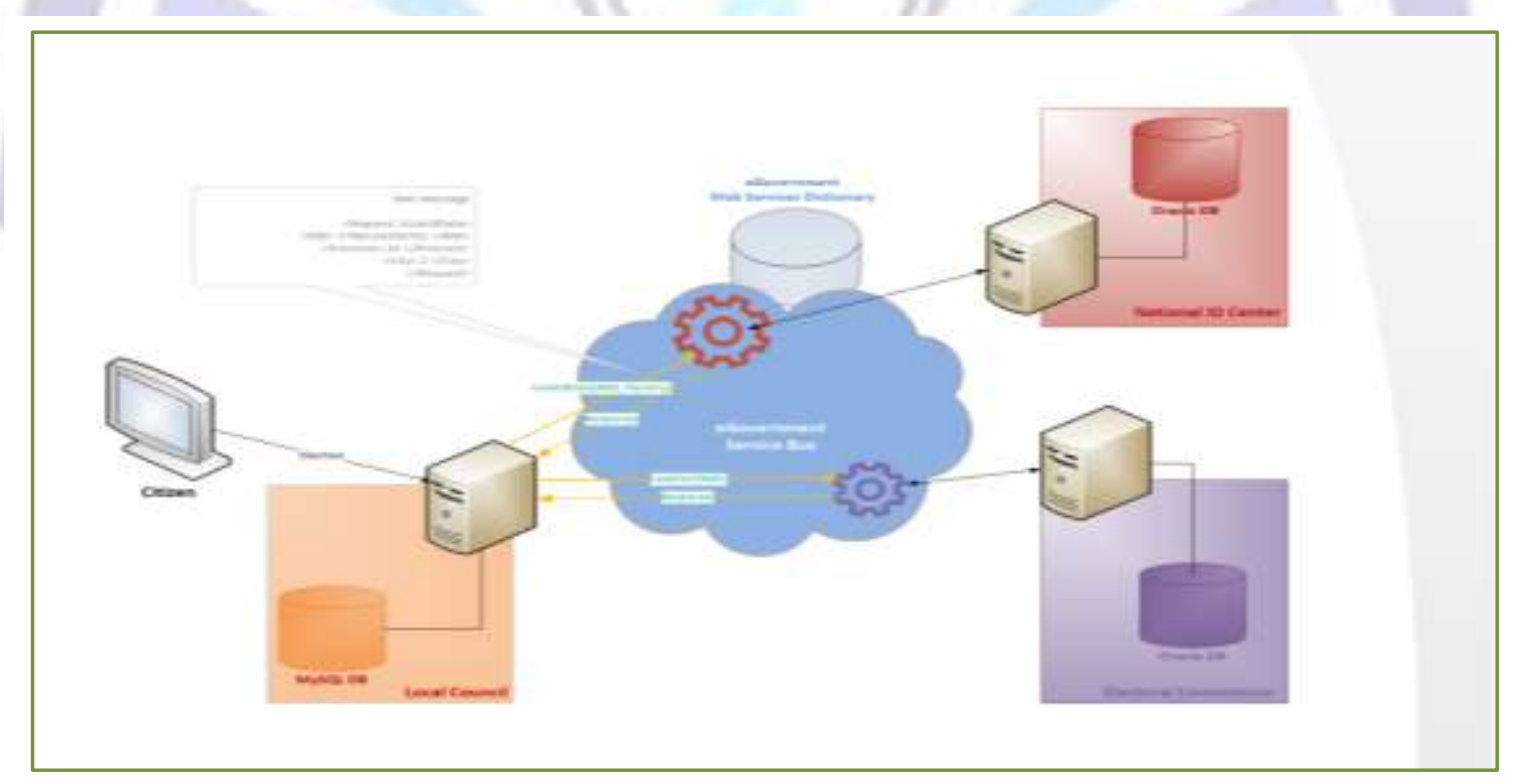

Fig (8) System integration in e-gov - level of DB 


\subsection{REQUIRMENTS FOR THE E-VOTING SYSTEM}

- Privacy: after casting a vote, no one should be able to link the voter to this vote;

- Authenticity: only eligible voters can cast their votes;

- Integrity/accuracy: once a voter cast a vote, no alternation to this vote is permitted. Moreover, all

- $\quad$ valid votes must be counted, whereas all invalid votes must not be counted;

- $\quad$ Security: throughout the voting process, a vote can't be tampered with;

- Democracy: all eligible voters must be able to vote, one person - one vote and no one can vote more than once or vote for others.

The system-specific requirements, on the other hand, are those requirements that are specific to the electronic-voting systems. The system-specific requirements include:

- Multi-user: a number of voters can vote simultaneously;

- Multi-campaign: a number of elections can be running simultaneously;

- Accessibility: the system can be accessed by voters from any location using secure Internet and/or

- mobile devices;

- $\quad$ Availability: the system must have high-availability during an election campaign.

\section{Case Study 2: Local Managerial Requests}

In this case an e-Government application is created based on the proposed framework in order to offer a web portal of Ismaillia Province. The web portal aims to enable and facilitate interaction between citizens and the local municipality of Ismaillia. Citizens are able to do some electronic requests for the following use-cases:

1. UC1: Work relocation request

2. UC2: Request for an apartment

The following figure shows the related activity diagram which represents the request process in the local province application.

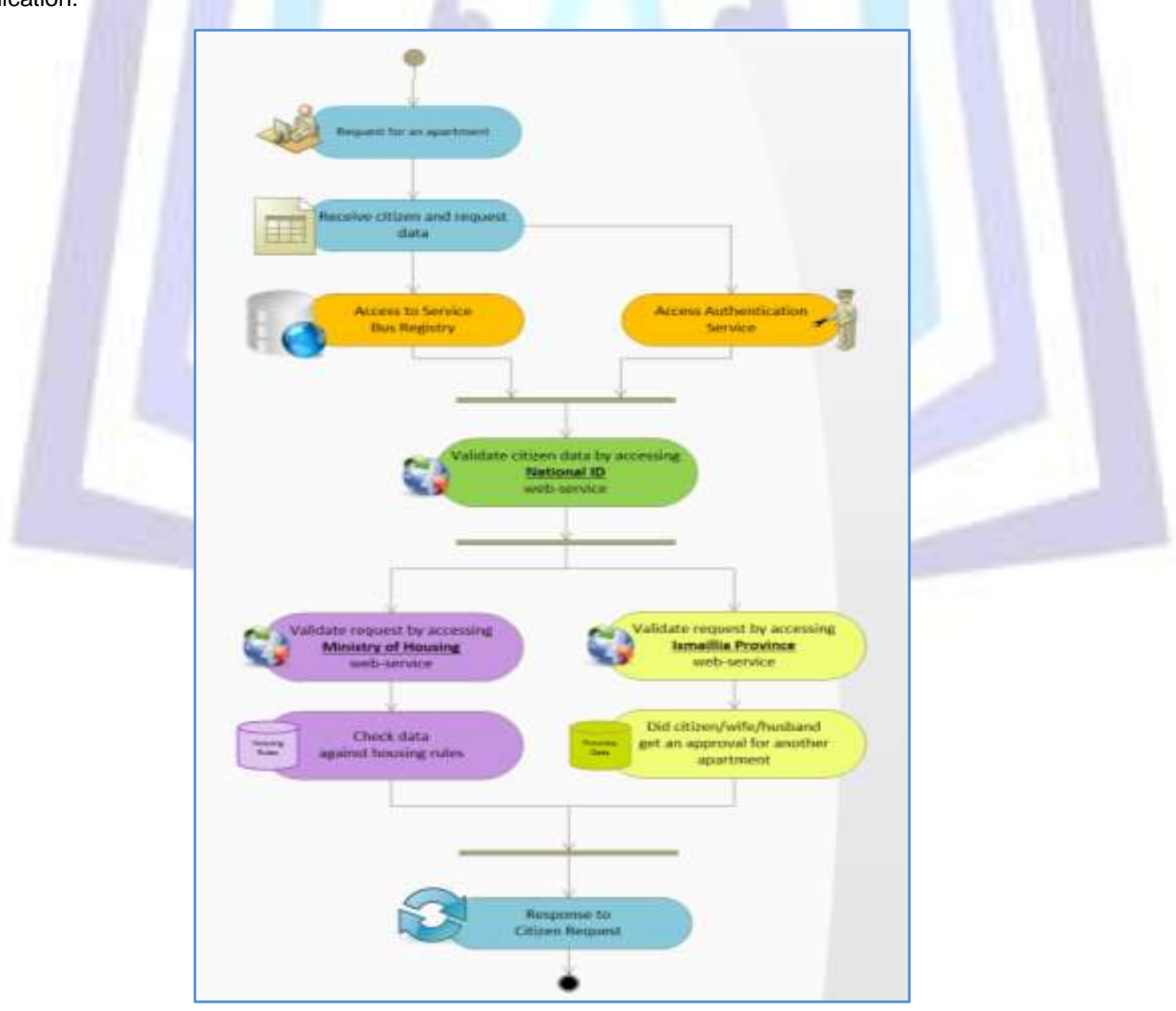

Fig (9) e-participation diagram 
The following figures show screenshots of the local services including

1. Transfer requests

2. Apartment request

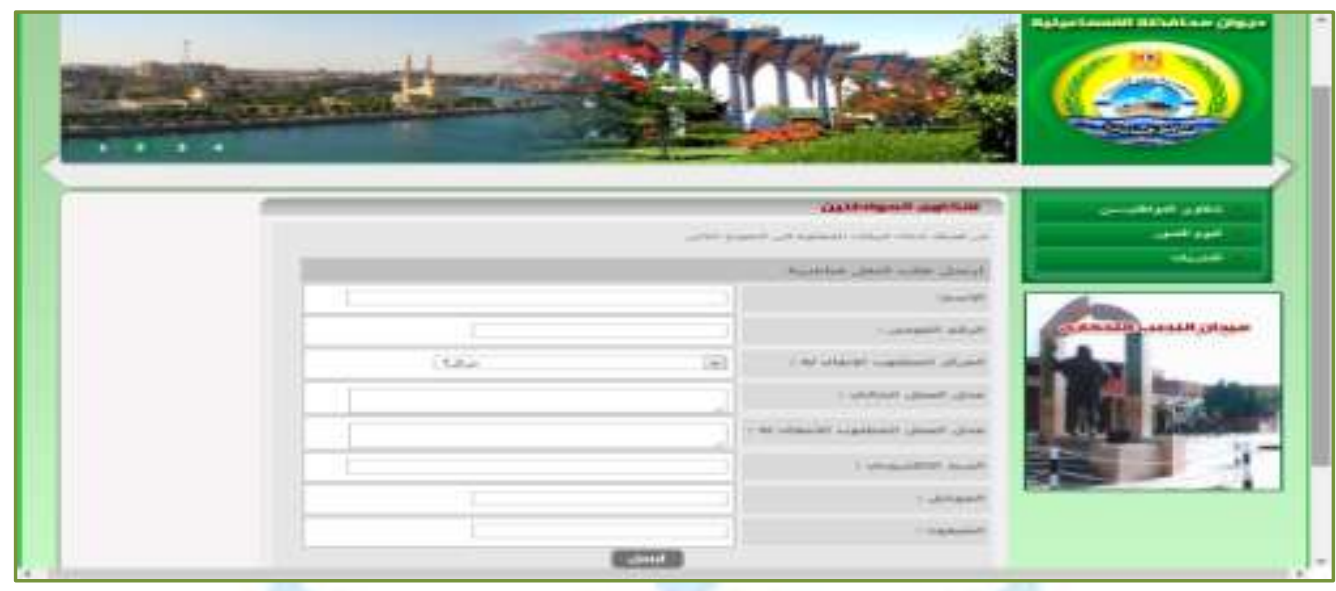

fig (10) application form for Transfer requests

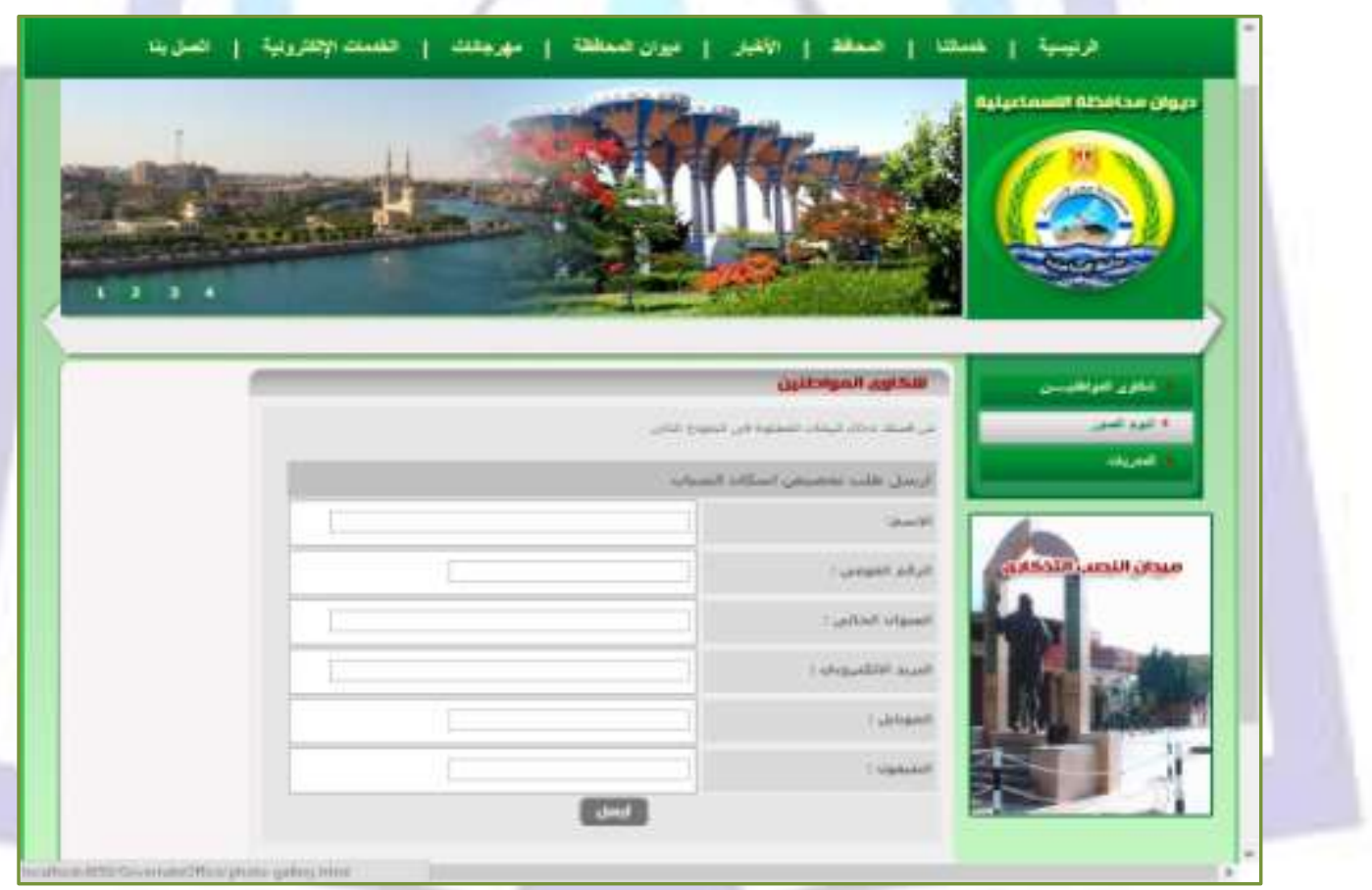

Fig (11) application form for Apartment request

\section{Framework Objectives}

Robust: This is defined as the resilience of the system when cheating behavior is detected, partial component or system malfunction occurs or when it is subjected to external malicious attacks. The system should operate as expected in abnormal conditions or in a hostile environment.

Flexible: The suggested framework must be able to support the changing nature of laws, rules and procedures without need to replace or modify any major features of e-Government systems.

Scalable: e-Government projects are usually scalable projects. They start by supporting few services and add functionalities to support the rest of services later. An e-Government framework must support the increasing supported services without modifying current services.

Integrated: The suggested framework must enable the integration between several e-Governmental applications and services in order to share and validate data, integrate procedures and simplify services. Integration capability must support easy and unexpected integration cases with minimum work. 
Heterogeneity: It aims to integrate heterogeneous e-Government applications based on different technologies and different data repositories. Knowing that e-Government applications are usually developed using several technologies and tools, "heterogeneity" becomes essential feature to enable "integration".

All these technical objectives allow to enhance the e-government project.

\section{Conclusions}

There are many results and conclusions for this paper they are:

- Based on the results of the study, the author therefore rejects the pessimistic view of e-democracy, which maintains that the application of ICT to enhance or transform the theory and practice of democracy is something of a luxury debate than a reality. Realising the dream of a perfected Athenian-style direct democracy may be a distant reality, yet countries like Egypt can instigate a staged implementation, which is quite possible and indeed feasible today. The researcher proposes an approach whereby the policy and decision makers explore the possibilities and opportunities of ICT to enhance, revitalise and transform democracy and to adopt countryspecific strategies and policies after consulting with stakeholders.

- $\quad$ one result of the research fundamentally enhances the knowledge of e-democracy in the Egypt context and sets the basis for further discussion of e-voting and e-democracy with a view to building an ideal and inclusive democracy for Egypt. The vision is clear, although it needs to be ratified through stakeholder consultation.

- This research started amidst the confusion and heated discussion surrounding the e-voting debate in Egypt; this has now provided a theoretical basis on which programs and projects can be planned and implemented before the debate starts again next elections.

- $\quad$ The proposed framework has approved solutions for the paper problems :

1-more engagement between citizens and government when using applications related to government parts web sites which enables citizens to participate, vote and assess people or process .

2-Poor of traditional democracy to achieve (accountability-transparency-social participation- evaluation of service delivery) can be resolved by depending on e-democracy concept.

3- Legacy systems and different technologies applied in many government agencies can interact with each other simply and securely. The paper Author has published a recent paper that provide a system that help Egyptian e-government can transform from second to third stage [3] ,the proposed framework can handle legacy systems integration which help in moving forward into the forth stage

4. Now Egyptian living abroad can participate in their country decision making If the government start evoting project .

The proposed framework has the following add values:

- Make it easier for business and individuals to deal with government.

- Improve communications between different parts of government so that people do not have to be asked repeatedly for the same information by different service providers.

- Give staff at call centers and other offices better access to information so that they can deal with members of the public more efficiently and more helpfully.

- Make it much easier for different parts of government to work in partnership: central government with local authorities or the voluntary sector; or government with third party delivery channels such as the Post Office or private sector companies.

\section{Recommendations}

1. e-voting systems implementation is preferred to in first level in Unions, universities and associations, i.e low level scale to build trust

2. second level e-voting can be used in municipals election until level of maturity

3. Recommendations for Egyptian e-government when implementing the proposed framework

\section{References}

1) Stephen Coleman \& Donald F. Norris, "A new agenda for e-democracy ",Oxford Internet Institute, Forum Discussion Paper No. 4, January 2005

2) URL,"http://www.w3schools.com"[accessed 1/06/2013].

3) Esam M. El Gohary and M. El-Sayed Wahed1," The suggested system for health insurance Application based on Smart Cards", IJCSI International Journal of Computer Science Issues, Vol. 10, Issue 1, January 2013 